\title{
Associação entre atividade física, aptidão cardiorrespiratória e biomarcadores inflamatórios em adultos jovens aparentemente saudáveis
}

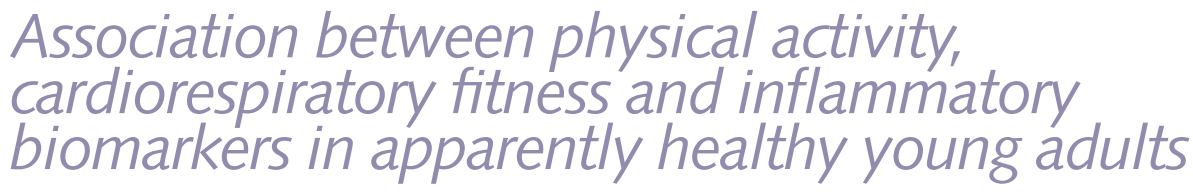

Carlos Marcelo Cuello Moreno ${ }^{1}$

Renata Bielemann ${ }^{2}$

Priscila M Nakamura ${ }^{2}$

Leony M Galliano ${ }^{1}$

Daniel D Bertoldi ${ }^{3}$

Martin Larangeira ${ }^{3}$

Helena S. Thurow ${ }^{4}$

Marcelo Mendonça ${ }^{4}$

Pedro Curi Hallal ${ }^{1,2}$

\section{Resumo}

A inflamação sistêmica crônica de baixa intensidade está relacionada com maior risco de doenças cardiovasculares. Estudos sugerem que a proteína C-reativa, um dos principais biomarcadores inflamatórios, pode estar inversamente relacionada com a prática de atividade física e com a aptidão física. $\mathrm{O}$ objetivo deste estudo transversal foi determinar as associações entre os níveis de atividade física e a aptidão cardiorrespiratória, com biomarcadores inflamatórios em homens adultos jovens (18-30 anos) aparentemente saudáveis $(\mathrm{N}=85)$. As amostras foram analisadas pelo método ELISA (Enzyme-Linked Immuno Sorbent Assay), usando kits de alta sensibilidade para proteína C-reativa, interleucina 6 , interleucina $1 \beta$ e TNF- $\alpha$. A prática de atividade física foi mensurada por questionário e acelerometria. $\mathrm{O}$ consumo máximo de oxigênio $\left(\mathrm{VO}_{2 \max }\right)$ foi estimado por teste incremental em cicloergômetro. $\mathrm{Na}$ análise bruta, a média das concentrações de proteína C-reativa da amostra foi de $1,59 \pm 1,16 \mathrm{mg} / \mathrm{L}$, e esteve significativamente correlacionada com o $\mathrm{VO}_{2 \max }(\mathrm{r}=-0,32 ; \mathrm{p}=0,03)$, mas não com atividade física medida por acelerometria ou questionário. $\mathrm{O}$ índice de massa corporal (IMC) e a circunferência abdominal apresentaram correlação significativa com a proteína C-reativa $(r=0,37 ; p<0,001$ e $r=0,41 ; p<0,001$, respectivamente). Quando incluímos no modelo o IMC e a circunferência da cintura, a aptidão física perdeu a significância. Não houve relação entre atividade física ou aptidão física com os demais marcadores inflamatórios. Conclui-se que nesse grupo de adultos jovens houve relação entre a proteína C-reativa e o $\mathrm{VO}_{2 \max }$, e que esta associação é explicada pelas modificações no perfil antropométrico decorrentes de altos níveis de aptidão cardiorrespiratória.

\section{Palavras-chave}

Inflamação; Proteína C-reativa; Atividade física; Aptidão física.

\begin{abstract}
Low-intensity chronic systemic inflammation is related to an increased risk of cardiovascular disease. Studies suggest that $C$-reactive protein, one of the main inflammatory biomarkers, may be inversely related to physical activity levels and physical fitness. The aim of this cross-sectional study was to evaluate the associations between physical activity, fitness and inflammatory biomarkers in apparently healthy men $(N=85)$ aged 18 to 30 years. $C$-reactive protein, interleukin 6 , interleukin $1 \beta$ and $T N F-\alpha$ were measured using the ELISA method. Physical activity practice was assessed by questionnaire and accelerometry. Maximum oxygen uptake $\left(V \mathrm{O}_{2 \max }\right)$ was estimated based on a cycle ergometer incremental test. In the unadjusted analysis, the mean concentration of $C$-reactive protein in the sample was $1.59 \pm 1.16 \mathrm{mg} / \mathrm{L}$, and was inversely correlated with $\mathrm{VO}_{2 \max }(r=-0.32, p=0.03)$, but not with physical activity estimated by accelerometry or questionnaire. Body mass index (BMI) and waist circumference also significantly correlate with C-reactive protein ( $r=0.37, p<0.001$ and $r=0.41, p<0.001$, respectively). After adjustment for anthropometric characteristics (BMI and waist circumference), the association with fitness was no longer significant. No association was observed between physical activity or fitness levels and the other inflammatory markers. We conclude that in this group of young adults, there was an inverse association between fitness and $C$-reactive protein, but this association is explained by the influence of fitness on anthropometry.
\end{abstract}

\section{Keywords}

Inflammation; C-Reactive Protein; Physical activity; Physical fitness.
Rev Bras Ativ Fis Saúde p. 501-510 DOl:

http://dx.doi.org/10.12820/rbafs.v.18n4p501

1 Programa de Pós-graduação em Educação Física - Universidade Federal de Pelotas

2 Programa de Pós-graduação em Epidemiologia - Universidade Federal de Pelotas

3 Curso de Biotecnologia - Universidade Federal de Pelotas

4 Programa de Pós-graduação em Biotecnologia - Universidade Federal de Pelotas 


\section{INTRODUÇÃO}

As doenças cardiovasculares e cerebrovasculares são as principais causas de mortes no mundo ${ }^{1}$. No Brasil, as doenças cardiovasculares atingem principalmente os sujeitos do sexo masculino ${ }^{2}$, e quando consideradas juntamente com diabetes, câncer e doenças respiratórias crônicas, representam a maior carga de morbimortalidade ${ }^{3}$. Tais enfermidades possuem um conjunto de fatores de risco comuns, incluindo tabagismo, inatividade física, alimentação inadequada e consumo excessivo de álcool ${ }^{4}$. Tendo em vista o alto custo social e financeiro que tais doenças representam para o sistema público de saúde, a prevenção torna-se essencial.

A inatividade física é responsável por $10 \%$ das mortes que ocorrem por ano no mundo ${ }^{5}$. Além de trazer benefícios fisiológicos, metabólicos e psicológicos, e reduzir o risco de doenças cardíacas, acidentes vasculares cerebrais, diabetes tipo $2 \mathrm{e}$ hipertensão arterial, a prática regular de atividades físicas está associada com a aptidão física, o controle de peso e a saúde mental ${ }^{6}$. O condicionamento cardiorrespiratório também tem um papel importante na prevenção de doenças vasculares e seus benefícios podem ser percebidos com pequenos aumentos dos níveis de aptidão ${ }^{7}$.

A inflamação sistêmica crônica de baixa intensidade, relacionada com algumas doenças vasculares, está também associada à inatividade física ${ }^{8}$. Existem alguns marcadores ou mediadores químicos que, além de determinar o grau da inflamação sistêmica, têm participação importante na aterogênese, processo de formação e desenvolvimento das placas ateroscleróticas nas paredes íntimas dos vasos arteriais. Entre os principais mediadores, também conhecidos por biomarcadores inflamatórios, estão a proteína C-reativa e algumas citocinas pró-inflamatórias, onde se destacam o fator de necrose tumoral alfa (TNF- $\alpha)$ e as interleucinas 6 (IL-6) e 1 beta (IL-1 $\beta)^{9,10}$.

Mesmo encontrando na literatura diversos estudos buscando esclarecer os efeitos anti-inflamatórios da prática regular de atividade física ou da aptidão aeróbia $^{11-20}$, ainda encontramos algumas lacunas no que se refere a este tema, principalmente em adultos jovens. Nesse contexto, o objetivo deste estudo foi determinar as associações entre os níveis de atividade física, a aptidão cardiorrespiratória e a força muscular, com biomarcadores inflamatórios em homens adultos jovens aparentemente saudáveis.

\section{METODOLOGIA}

Este estudo foi realizado em caráter observacional com desenho transversal e aprovado pelo Comitê de Ética da Faculdade de Medicina da Universidade Federal de Pelotas (UFPEL), sob o protocolo CAAE número 07477612.9.0000.5317. A amostra foi constituída por 85 homens adultos jovens, com idade entre 18 e 30 anos, residentes na zona urbana da cidade de Pelotas-RS, recrutados por conveniência. A inclusão somente de sujeitos do sexo masculino na amostra se justifica por esta ser a população que apresenta maior risco para eventos cardiovasculares ${ }^{2}$. Para participarem do estudo, os indivíduos deveriam apresentar condições físicas para a realização de um teste cardiorrespiratório de esforço máximo. Além disso, não foram incluídos sujeitos com hipertensão arterial, diabetes ou cardiopatias e que estivessem sob efeito de medicação farmacológica que pudesse influenciar nas concentrações dos biomarcadores inflamatórios (por exemplo, corticosteróides, anti-inflamatórios, estatinas). 
Para o cálculo de diferença mínima detectável considerou-se um poder fixo de $80 \%$, com prevalência de ativos no lazer nesta faixa etária de $35 \%{ }^{21}$ e desvio-padrão da média geométrica de proteína C-reativa de $3,21 \mathrm{mg} / \mathrm{L}^{22}$, buscando detectar uma diferença mínima de 1,88 mg/L na média geométrica comparando indivíduos ativos e inativos.

Os dados foram coletados dentro de um período de treze dias consecutivos, com duas visitas de cada participante ao Laboratório de Bioquímica e Fisiologia do Exercício da Escola Superior de Educação Física (ESEF) da UFPEL. Através de uma ligação telefônica foi realizado o recrutamento e agendamento dos sujeitos da amostra. No primeiro dia de visita ao laboratório foi aplicado um questionário geral, por meio de entrevista, contendo questões de dados pessoais de identificação, características demográficas e possíveis fatores de confusão. Depois foram realizadas as medidas antropométricas (peso corporal, estatura e circunferência abdominal) que foram avaliadas por uma balança eletrônica (precisão de $0,1 \mathrm{Kg}$ ) da marca Filizola, um estadiômetro de parede e fita métrica flexível e inextensível da marca Sanny, respectivamente. Neste dia também foi realizada a coleta sanguínea, por uma enfermeira treinada, assim como a centrifugação e armazenamento do material coletado. Por último, com o objetivo de determinar o nível de atividade física de maneira objetiva, cada um dos participantes recebeu um acelerômetro ativado, assim como, as instruções e recomendações sobre a sua utilização.

$\mathrm{Na}$ segunda visita ao laboratório, os participantes efetuaram a devolução dos acelerômetros. Logo em seguida, foram submetidos a um questionário com a finalidade de estimar o nível de prática habitual de atividade física e o comportamento sedentário. Após, os indivíduos realizaram um teste para determinar a força muscular por meio da força de preensão manual, utilizando um dinamômetro digital da marca Jamar. A seguir foi realizado um teste incremental em cicloergômetro para estimar a aptidão cardiorrespiratória.

Para determinar os níveis de atividade física foram utilizados dois diferentes métodos: questionário e acelerometria. O questionário foi o International Physical Activity Questionnaire (IPAQ), versão longa, por meio de entrevista, estimando os últimos 7 dias $^{23}$. Utilizou-se também um questionário para estimar o comportamento sedentário, desenvolvido em projeto concomitante conduzido por outro aluno de pós-graduação ${ }^{24}$. O instrumento avalia o tempo despendido em atividades realizadas na posição sentada durante o tempo de lazer, no deslocamento para o trabalho e no trabalho.

Para a medida objetiva dos níveis de atividade física foi utilizado um acelerômetro triaxial da marca Actigraph modelo GT1M, com epoch de 5 segundos. Para a ativação do aparelho e, para o armazenamento e processamento das informações, utilizamos o programa Actilife 4.6.1. O acelerômetro foi utilizado na cintura por seis dias consecutivos, sendo que o primeiro e o último dia de uso, por serem incompletos, foram excluídos da análise.

O protocolo para a estimação da aptidão cardiorrespiratória, consumo máximo de oxigênio $\left(\mathrm{VO}_{2 \max }\right)$ consistiu de um teste incremental até a exaustão realizado em um cicloergômetro da marca Ergo-Cycle. $\mathrm{O}$ momento de exaustão foi definido quando o indivíduo não conseguia manter a carga por um período de 1 minuto, desistência voluntária ou BORG igual a 20. As cargas aumentaram da seguinte forma: uma carga de 50 watts por 3 minutos de aquecimento; após, a carga foi aumentada para 100 watts, sendo que, a cada 2 minutos, a carga foi aumentada em 50 watts $^{25}$. Por motivo de segurança e controle, durante o teste de esforço foram monitoradas as respostas hemodinâmicas através das medidas de pressão arterial e frequência cardíaca, além da percepção subjetiva de esforço pela escala de 14 etapas (6-20) de Borg. 
A força de preensão manual foi determinada utilizando um dinamômetro digital da marca Jamar. Cada indivíduo realizou três esforços máximos com cada mão. Os testes ocorreram com os participantes sentados em uma cadeira, com os ombros aduzidos, cotovelos flexionados em 90 graus e o pulso em posição neutra. A maior medida das seis tentativas foi utilizada nas análises.

No contato telefônico de recrutamento e agendamento, os indivíduos foram instruídos sobre o jejum prévio obrigatório de no mínimo oito horas para a coleta de sangue, e, foram orientados a evitarem o consumo de álcool no dia anterior. Após a coleta de $6 \mathrm{~mL}$ de sangue, através de um procedimento a vácuo, as amostras foram preparadas em tubos de separação de soro sem anticoagulante e contendo gel separador e ativador de coágulo e mantidas à temperatura ambiente durante 30 minutos para coagular. Após centrifugar, a $3500 \mathrm{rpm}$ durante 15 minutos, o soro foi dividido em duas alíquotas ( $2 \mathrm{ml}$ de soro) e armazenado a $-80^{\circ} \mathrm{C}$ para as análises posteriores no Laboratório de Genômica Funcional do Centro de Desenvolvimento Tecnológico da UFPEL. As amostras foram analisadas pelo método de ELISA (Enzyme-Linked Immuno Sorbent Assay), usando kits de alta sensibilidade adquiridos da Invitrogen (ELISA; Invitrogen, Camarillo, $C A$ ) para proteína C-reativa (\#KHA0031), IL-6 (\#KHC0061), IL-1 $\beta$ (\#KHC0014) e TNF- $\alpha$ (\#KAC1751). Todas as amostras foram analisadas em duplicatas e a média dos dois valores foi utilizada para a análise de dados. As absorbâncias do ELISA foram medidas a 450 $\mathrm{nm}$, utilizando o leitor de microplacas Victor X5 (PerkinElmer, Waltham, MA). As concentrações foram determinadas traçando um gráfico dos dados de absorbância dos padrões com as concentrações normais, conforme as instruções do fabricante.

A análise estatística incluiu uma descrição da amostra, com cálculos de média e desvio-padrão para as variáveis numéricas. Logo após foi confirmada a distribuição normal das variáveis principais em estudo por inspeção visual. A seguir, foi procedida a correlação de Pearson entre as medidas dos marcadores inflamatórios e as variáveis independentes. Logo após, foi realizada análise de variância, na qual foram comparadas as médias dos marcadores inflamatórios entre as categorias das variáveis independentes. Por fim, rodou-se um modelo de regressão linear, utilizando a proteína C-reativa como desfecho. Para tais análises foi utilizado o pacote estatístico STATA 12.0, considerando um nível de significância de 5\%.

\section{RESULTADOS}

As principais características dos indivíduos estudados podem ser visualizadas na tabela 1. A média de idade dos participantes foi de 22,7 $\pm 2,8$ anos. Houve ampla heterogeneidade da amostra no que se refere ao índice de massa corporal (IMC) ( média $24,9 \pm 3,2 \mathrm{Kg} / \mathrm{m}^{2}$ ) e circunferência abdominal (média $81,4 \pm 8,3 \mathrm{~cm}$ ). Os níveis de atividade física determinados pelo acelerômetro apresentaram as médias de 60,2 $\pm 25,2$ $\mathrm{min} /$ dia de atividades físicas de intensidade moderada e 6,3 $\pm 7,9 \mathrm{~min} /$ dia de atividades física de intensidade vigorosa. Já o questionário mostrou que a atividade física total no lazer teve uma média de 408,8 $\$ 365,0 \mathrm{~min} / \mathrm{sem}$, o equivalente a $58,4 \mathrm{~min} / \mathrm{dia}$, similar ao observado com o acelerômetro. Em relação ao comportamento sedentário, o tempo despendido somente assistindo televisão ou no computador teve média de $307,4 \pm 207,1 \mathrm{~min} /$ dia. A média do valor estimado de $\mathrm{VO}_{2 \max }$ foi de $41,9 \pm 7,1 \mathrm{~mL} . \mathrm{Kg}^{-1}$. $\min ^{-1}$. A força de preensão manual apresentou o valor médio de $47,7 \pm 7,9 \mathrm{Kg} / \mathrm{f}$.

A média encontrada das concentrações de proteína $C$-reativa entre os sujeitos da amostra $(\mathrm{n}=85)$ foi de $1,59 \pm 1,16 \mathrm{mg} / \mathrm{L}$. Os valores para IL-6, IL-1 $\beta$ e TNF- $\alpha$ foram $1,42 \pm 2,56 \mathrm{pg} / \mathrm{mL}, 0,16 \pm 0,06 \mathrm{pg} / \mathrm{mL}$ e $1,08 \pm 0,17 \mathrm{pg} / \mathrm{mL}$, respectivamente. 
Tabela 1 - Características da amostra e variáveis de exposição e desfecho $(n=85)$.

\begin{tabular}{lccc}
\hline \multicolumn{1}{c}{ Variáveis } & Média & DP & Mínimo - Máximo \\
\hline Idade (anos) & 22,65 & 2,82 & $18,00-30,00$ \\
\hline IMC (Kg/m²) & 24,88 & 3,21 & $17,96-34,27$ \\
\hline Circunferência abdominal (cm) & 81,37 & 8,26 & $64,00-108,5$ \\
\hline AF moderada - Ac (min/dia) & 60,24 & 25,19 & $16,00-133,50$ \\
\hline AF vigorosa - Ac (min/dia) & 6,25 & 7,90 & $0,00-32,33$ \\
\hline AF moderada e vigorosa - Ac (min/dia) & 67,41 & 29,66 & $16,50-145,67$ \\
\hline AF lazer - IPAQ (min/sem) & 408,80 & 364,95 & $0,00-630,00$ \\
\hline AF vigorosa - IPAQ (min/sem) & 154,42 & 154,88 & $30,00-3790,00$ \\
\hline Tempo de tela (min/dia) & 307,40 & 207,09 & $25,71-985,71$ \\
\hline VO ${ }_{2 m a x}$ (mL.Kg-1.min-1) & 41,92 & 7,14 & $27,58-65,13$ \\
\hline Força de preensão manual (Kg/f) & 47,68 & 7,93 & $30,90-73,90$ \\
\hline Proteína C-reativa (mg/L) & 1,59 & 1,16 & $0,02-3,63$ \\
\hline IL-6 (pg/mL) & 1,42 & 2,56 & $0,15-18,85$ \\
\hline IL-1 $\beta$ (pg/mL) & 0,16 & 0,06 & $0,13-0,63$ \\
\hline TNF- $\alpha$ (pg/mL) & 1,08 & 0,17 & $0,85-1,91$ \\
\hline
\end{tabular}

IMC - índice de massa corporal; AF - atividade física; Ac - acelerômetro; IPAQ - International Physical Activity Questionnaire; $\mathrm{VO}_{2 \max }$ - consumo máximo de oxigênio; IL-6 - Interleucina-6; IL-1 $\beta$ - Interleucina-1beta; TNF- $\alpha$ - fator de necrose tumoral alfa.

A tabela 2 apresenta a correlação entre os marcadores inflamatórios e as variáveis antropométricas. Houve correlações significativas da proteína C-reativa com o IMC ( $r=0,37 ; p<0,001)$, circunferência abdominal $(r=0,41 ; p<0,001)$ e $\mathrm{VO}_{2 \max }$ $(r=-0,32 ; p=0,03)$. Baixos e não significativos valores de correlação foram observados com os níveis de atividade física, estimados tanto por questionário quanto por acelerometria, e com a força de preensão manual. Não houve correlações com os outros biomarcadores inflamatórios.

$\mathrm{Na}$ análise de variância (tabela 3) comparando as médias dos biomarcadores entre as categorias das variáveis independentes, os resultados foram semelhantes. Novamente, $\mathrm{o} \mathrm{VO}_{2 \max }$ foi o indicador que apresentou relação significativa com a proteína C-reativa. Indivíduos com $\mathrm{VO}_{2 \max }$ acima de $42,0 \mathrm{~mL} \cdot \mathrm{Kg}^{-1} \cdot \mathrm{min}^{-1}$ tiveram um valor médio de $0,67 \mathrm{mg} / \mathrm{L}$ inferior àqueles com valores abaixo de 42,0 $\mathrm{mL} \cdot \mathrm{Kg}^{-1} \cdot \mathrm{min}^{-1}$. A circunferência da cintura também esteve relacionada fortemente com a proteína C-reativa. A diferença entre os indivíduos com circunferência da cintura acima de $94 \mathrm{~cm}$ e aqueles com valores inferiores a esse foi de $1,37 \mathrm{mg} / \mathrm{L}$. O IMC também esteve associado com os valores de proteína $\mathrm{C}$-reativa, embora com menor magnitude da associação. Novamente, os demais marcadores inflamatórios não estiveram associados às exposições.

$\mathrm{Na}$ tabela 4 realizamos uma regressão linear com três modelos analisando os efeitos do $\mathrm{VO}_{2 \max }$ sobre a proteína C-reativa. No modelo 1 a análise bruta mostrou que, o incremento de $1,0 \mathrm{~mL} \cdot \mathrm{Kg}^{-1} \cdot \mathrm{min}^{-1}$ no $\mathrm{VO}_{2 \max }$ diminuiu $0,05 \mathrm{mg} / \mathrm{L}$ na concentração do marcador inflamatório (IC95\% de -0,08 a -0,02; p=0,003). Porém, quando realizamos a análise ajustando para as variáveis antropométricas, o $\mathrm{VO}_{2 \max }$ deixou de estar estatisticamente relacionado, sugerindo que a influência da aptidão cardiorrespiratória sobre o biomarcador passa diretamente pelo seu efeito sobre as variáveis antropométricas. Já a circunferência abdominal e o IMC continuaram tendo associação estatisticamente significativa com a proteína C-reativa mesmo ajustando para $\mathrm{VO}_{2 \max }$. 
Tabela 2 - Correlações entre marcadores inflamatórios e as variáveis antropométricas e de aptidão física.

\begin{tabular}{lccccccccc}
\hline \multirow{2}{*}{ Variáveis } & \multicolumn{2}{c}{$\begin{array}{c}\text { Proteína } \\
\text { C-reativa }\end{array}$} & \multicolumn{2}{c}{ IL-1 $\beta$} & & IL-6 & \multicolumn{2}{c}{ TNF- $\alpha$} \\
\cline { 2 - 10 } & \multicolumn{1}{c}{$r$} & $\mathrm{p}$ & $\mathrm{r}$ & $\mathrm{P}$ & $\mathrm{r}$ & $\mathrm{p}$ & $\mathrm{r}$ & $\mathrm{p}$ \\
\hline IMC & 0,31 & 0,004 & $-0,07$ & 0,50 & 0,0004 & 1,0 & $-0,004$ & 0,97 \\
\hline Circunferência abdominal & 0,41 & $<0,001$ & 0,005 & 0,96 & 0,05 & 0,65 & 0,18 & 0,11 \\
\hline AF moderada e vigorosa - Ac & 0,05 & 0,65 & $-0,02$ & 0,89 & $-0,05$ & 0,68 & $-0,10$ & 0,35 \\
\hline AF vigorosa - Ac & 0,06 & 0,58 & $-0,04$ & 0,70 & $-0,13$ & 0,23 & $-0,25$ & 0,02 \\
\hline AF total de lazer - IPAQ & 0,09 & 0,37 & 0,04 & 0,74 & $-0,03$ & 0,76 & $-0,08$ & 0,45 \\
\hline AF vigorosa de lazer - IPAQ & 0,10 & 0,35 & 0,03 & 0,76 & $-0,03$ & 0,81 & $-0,08$ & 0,47 \\
\hline Tempo de tela & 0,11 & 0,33 & 0,09 & 0,41 & 0,04 & 0,72 & 0,03 & 0,76 \\
\hline VO ${ }_{2 \text { max }}$ & $-0,32$ & 0,003 & 0,006 & 0,96 & $-0,08$ & 0,45 & $-0,19$ & 0,09 \\
\hline Força de preensão manual & 0,05 & 0,68 & $-0,09$ & 0,42 & 0,05 & 0,68 & 0,04 & 0,73 \\
\hline
\end{tabular}

IMC - índice de massa corporal; AF - atividade física; Ac - acelerômetro; IPAQ - International Physical Activity Questionnaire; $\mathrm{VO}_{2 \max }$ - consumo máximo de oxigênio.

Tabela 3 - Análise de variância comparando a média dos marcadores inflamatórios entre as categorias das variáveis antropométricas e de aptidão física.

\begin{tabular}{|c|c|c|c|c|c|c|c|c|}
\hline & \multicolumn{2}{|c|}{ Proteína C-reativa } & \multicolumn{2}{|c|}{ IL-1 $\beta$} & \multicolumn{2}{|c|}{ IL-6 } & \multicolumn{2}{|c|}{ TNF- $\alpha$} \\
\hline & Média (dp) & p & Média (dp) & p & Média (dp) & p & Média (dp) & p \\
\hline IMC $\left(\mathrm{Kg} / \mathrm{m}^{2}\right)$ & & 0,04 & & 0,60 & & 0,36 & & 0,83 \\
\hline$\geq 25$ & $1,86(1,22)$ & & $0,16(0,01)$ & & $1,70(3,24)$ & & $1,07(0,13)$ & \\
\hline$<25$ & $1,35(1,06)$ & & $0,17(0,09)$ & & $1,17(1,74)$ & & $1,09(0,21)$ & \\
\hline Circunferência abdominal (cm) & & 0,009 & & 0,54 & & 0,18 & & 0,76 \\
\hline$\geq 94$ & $2,88(0,87)$ & & $0,15(0,01)$ & & $0,70(0,55)$ & & $1,10(0,14)$ & \\
\hline$<94$ & $1,51(1,13)$ & & $0,17(0,06)$ & & $1,46(2,63)$ & & $1,08(0,18)$ & \\
\hline AF moderada e vigorosa - Ac & & 0,42 & & 0,62 & & 0,85 & & 0,83 \\
\hline Tercil superior & $1,74(1,14)$ & & $0,16(0,01)$ & & $1,34(2,21)$ & & $1,06(0,12)$ & \\
\hline Tercis 1 e 2 & $1,52(1,17)$ & & $0,17(0,07)$ & & $1,45(2,72)$ & & $1,08(0,20)$ & \\
\hline$A F$ vigorosa $-A c$ & & 0,25 & & 0,55 & & 0,83 & & 0,04 \\
\hline Tercil superior & $1,38(0,98)$ & & $0,16(0,01)$ & & $1,33(2,17)$ & & $1,02(0,12)$ & \\
\hline Tercis 1 e 2 & $1,69(1,23)$ & & $0,17(0,07)$ & & $1,46(2.75)$ & & $1,11(0,19)$ & \\
\hline AF total de lazer - IPAQ (min/sem) & & 0,21 & & 0,97 & & 0,36 & & 0,31 \\
\hline$\geq 150$ & $1,69(1,13)$ & & $0,17(0,07)$ & & $1,56(3,02)$ & & $1,06(0,15)$ & \\
\hline$<150$ & $1,34(1,19)$ & & $0,16(0,01)$ & & $1,07(0,56)$ & & $1,12(0,23)$ & \\
\hline AF vigorosa de lazer - IPAQ & & 0,81 & & 0,45 & & 0,47 & & 0,24 \\
\hline Tercil superior & $1,63(1,12)$ & & $0,18(0,10)$ & & $2,35(4,31)$ & & $1,04(0,13)$ & \\
\hline Tercis 1 e 2 & $1,57(1,19)$ & & $0,16(0,01)$ & & $0,96(0,46)$ & & $1,10(0,19)$ & \\
\hline Tempo de tela & & 0,38 & & 0,52 & & 0,68 & & 0,60 \\
\hline Tercil superior & $1,76(1,15)$ & & $0,15(0,01)$ & & $0,97(0,44)$ & & $1,06(0,15)$ & \\
\hline Tercis 1 e 2 & $1,52(1,16)$ & & $0,17(0,07)$ & & $1,61(3,02)$ & & $1,08(0,19)$ & \\
\hline $\mathrm{VO}_{2 \max }\left(\mathrm{mL} \cdot \mathrm{Kg}^{-1} \cdot \mathrm{min}^{-1}\right)$ & & 0,007 & & 0,55 & & 0,31 & & 0,07 \\
\hline$\geq 42$ & $1,28(1,10)$ & & $0,16(0,04)$ & & $1,53(3,13)$ & & $1,03(0,13)$ & \\
\hline$<42$ & $1,95(1,14)$ & & $0,17(0,07)$ & & $1,28(1,69)$ & & $1,10(0,19)$ & \\
\hline Força de preensão manual & & 0,78 & & 0,18 & & 0,73 & & 0,83 \\
\hline Tercil superior & $1,54(1,25)$ & & $0,16(0,05)$ & & $1,59(3,25)$ & & $1,08(0,19)$ & \\
\hline Tercis 1 e 2 & $1,61(1,11)$ & & $0,17(0,07)$ & & $1,32(2,09)$ & & $1,08(0,17)$ & \\
\hline
\end{tabular}

IMC - índice de massa corporal; AF - atividade física; Ac - acelerômetro; IPAQ - International Physical Activity Questionnaire; VO ${ }_{2 \text { max }}-$ con- $^{-}$ sumo máximo de oxigênio. 
Tabela 4 - Análise de regressão linear entre a proteína $\mathrm{C}$-reativa e $\mathrm{VO}_{2 \max }$ (consumo máximo oxigênio) e variáveis antropométricas em adultos jovens aparentemente saudáveis do sexo masculino.

\begin{tabular}{|c|c|c|c|c|c|c|c|c|c|}
\hline \multirow{2}{*}{ Variáveis } & \multicolumn{3}{|c|}{ Modelo 1} & \multicolumn{3}{|c|}{ Modelo 2} & \multicolumn{3}{|c|}{ Modelo 3} \\
\hline & $\beta$ & $I C_{95 \%}$ & $\mathrm{p}$ & $\beta$ & $I C_{95 \%}$ & $p$ & $\beta$ & $I C_{95 \%}$ & $p$ \\
\hline $\begin{array}{l}\mathrm{VO}_{2} \max \\
\left(\mathrm{mL} \cdot \mathrm{Kg}^{-1} \cdot \mathrm{min}^{-1}\right)\end{array}$ & $-0,05$ & $-0,08 ;-0,02$ & 0,003 & $-0,02$ & $-0,06 ; 0,02$ & 0,4 & $-0,02$ & $-0,06 ; 0,01$ & 0,2 \\
\hline $\begin{array}{l}\text { Circunferência } \\
\text { abdominal }(\mathrm{cm})\end{array}$ & 0,06 & 0,$03 ; 0,09$ & $<0,001$ & 0,05 & 0,$01 ; 0,09$ & 0,007 & - & --- & - \\
\hline $\begin{array}{l}\text { IMC } \\
\left(\mathrm{Kg} / \mathrm{m}^{2}\right)\end{array}$ & 0,13 & 0,$06 ; 0,21$ & 0,001 & - & --- & - & 0,10 & 0,$01 ; 0,19$ & 0,03 \\
\hline
\end{tabular}

IMC - Índice de massa corporal; Modelo 1: Análise bruta; Modelo 2: $\mathrm{VO}_{2 \max }+$ circunferência abdominal; $M o d e l o ~ 3: \mathrm{VO}_{2 \max }+\mathrm{IMC}$.

\section{DISCUSSÃO}

A inflamação crônica de baixa intensidade, identificada por altas concentrações de alguns mediadores químicos e de proteínas de fase aguda, vem sendo tema de muitos trabalhos relacionados à saúde. $\mathrm{Na}$ literatura podemos observar diversos estudos mostrando que esse quadro pode estar associado a alguns hábitos de vida, como, por exemplo, a prática de atividades físicas ${ }^{13-16}$. Em nosso estudo, o $\mathrm{VO}_{2 \max }$, a circunferência da cintura e o IMC estiveram fortemente associados à proteína C-reativa, sendo que o efeito do $\mathrm{VO}_{2 \max }$ foi explicado por suas consequências sobre tais indicadores antropométricos.

Estudos observacionais demonstraram que a prática de atividade física e o VO${ }_{2 \max }$ estão associados com os mediadores inflamatórios nas diferentes etapas da vida das pessoas ${ }^{18-20}$. Por outro lado, alguns estudos não apresentaram os mesmos resultados, e outros ainda não mostraram a mesma associação quando seus resultados foram ajustados para alguns fatores de confusão ${ }^{22,26,27}$. Uma série de outros fatores pode afetar a associação entre atividade física, $\mathrm{VO}_{2 \max }$ e marcadores inflamatórios, como as diferenças nas características dos sujeitos, o momento das coletas de sangue, diferentes ensaios laboratoriais para medir os marcadores inflamatórios, tabagismo, entre outros. Em relação à atividade física, as discrepâncias podem ser explicadas também pelos diferentes métodos de mensuração e pontos de corte para determinar se o indivíduo é ativo ou não ${ }^{28}$.

No presente estudo não encontramos diferenças significativas da proteína C-reativa em relação aos níveis de atividade física corroborando com Nazmi e colaboradores $^{26}$, em estudo realizado com 3.289 indivíduos brasileiros, adultos jovens (1.919 homens e 1.370 mulheres) com 22-24 anos de idade, também usando o IPAQ. Um fato importante que deve ser considerado neste caso é a faixa etária, pois sujeitos jovens aparentemente saudáveis tendem a apresentar baixas concentrações de biomarcadores inflamatórios. No entanto, um estudo populacional prospectivo encontrou resultados diferentes. Analisando 13.748 adultos norte-americanos com 20 anos de idade ou mais, observou-se que quanto maior o nível de atividade física relatada (leve, moderada ou vigorosa), menores eram os níveis de proteína C-reativa, em comparação com nenhuma atividade física ${ }^{12}$.

Entre as pesquisas que avaliaram a associação entre proteína C-reativa e o $\mathrm{VO}_{2 \max }$, a maioria das publicações demonstrou a relação inversa ${ }^{11,13,17}$. Church e colaboradores ${ }^{11}$ analisaram 722 homens brancos, com a idade entre 40 e 60 anos, participantes do estudo norte-americano concluindo que a proteína C-reativa plasmática foi inversamente relacionada com capacidade aeróbia $(\mathrm{p}<0,001)$. Diferentemente do nosso estudo, os autores encontraram que a associação se manteve 
mesmo após ajuste para o percentual de gordura corporal e a circunferência da cintura. Com as mesmas características, pesquisadores norte-americanos avaliaram 170 homens, com idade média de $51 \pm 9,3$ anos, e constataram uma relação inversa entre o $\mathrm{VO}_{2 \max }$ (determinado em teste de esforço máximo em esteira com medida direta) e a proteína C-reativa. Os resultados também foram ajustados para idade, IMC, fatores de risco de doenças coronárias e variáveis de estilo de vida ${ }^{17}$.

Um estudo israelita desenvolvido por Aronso e colaboradores ${ }^{13}$ verificou a relação da capacidade aeróbia e proteína C-reativa em 1.640 indíviduos (1.060 homens e 580 mulheres), com a média de idade de $50 \pm 10$ anos, divididos em três grupos: sujeitos com síndrome metabólica (três ou mais anormalidades metabólicas), sujeitos com uma ou duas anormalidades metabólicas, e sujeitos normais metabolicamente. A aptidão cardiorrespiratória foi determinada através de um teste incremental em esteira com medida indireta utilizando o protocolo de Bruce. Foi encontrada uma forte relação inversa entre capacidade aeróbia e proteína C-reativa $(\mathrm{p}=0,01)$.

Chama atenção o fato de que os estudos que constataram diferenças significativas, em sua maioria, foram realizados em populações de meia-idade e idosos. Nessa faixa etária, normalmente, os níveis de inflamação crônica de baixa intensidade e seus marcadores são maiores e facilmente detectáveis. Esse fato justifica nosso interesse em realizar este estudo com sujeitos jovens, visto que em idades mais avançadas existe clareza na associação entre aptidão física e marcadores inflamatórios. A escolha da população jovem trouxe algumas dificuldades analíticas. Por exemplo, os biomarcadores TNF- $\alpha, \mathrm{IL}-1 \beta$ e IL- 6 foram pouco heterogêneos, gerando ausência de associação com quaisquer indicadores de atividade física ou variáveis antropométricas.

Conclui-se que nesse grupo de adultos jovens houve relação entre a proteína C-reativa e o $\mathrm{VO}_{2 \max }$ estimado, e que esta associação é explicada pelas modificações no perfil antropométrico decorrentes dos altos níveis de aptidão cardiorrespiratória. A prática de atividade física, medida por acelerometria ou questionário, não se relacionou com a proteína C-reativa ou com os demais marcadores inflamatórios estudados.

\section{Contribuições dos autores}

CM Moreno teve a ideia original e participou de todas as fases do estudo, desde sua concepção até a escrita. R Bielemann, PM Nakamura e LM Galliano coordenaram a coleta dos dados, auxiliaram na elaboração dos protocolos e interpretação dos dados. DD Bertoldi e M Laranjeira foram responsáveis pelo manejo das amostras biológicas e auxiliaram na análise do material biológico coletado. $\mathrm{H}$ Thurow e M Mendonça planejaram o protocolo de coleta do material biológico e conduziram a análise do material. PC Hallal supervisionou todas as fases do trabalho. Todos os autores participaram da escrita do artigo, dando contribuições intelectuais para o aprimoramento do mesmo. Todos os autores aprovaram a versão submetida para publicação.

\section{Comitê de Ética}

Todos os indivíduos assinaram um termo de consentimento livre e esclarecido concordando com a participação do estudo, que foi aprovado pelo comitê de ética da Faculdade de Medicina da Universidade Federal de Pelotas sob o protocolo CAAE número 07477612.9.0000.5317. 


\section{REFERÊNCIAS}

1. World Health Organization. Noncommunicable Diseases and Mental Health, World Health Organization. In Integrated management of cardiovascular risk: report of a WHO meeting, Geneva 9-12, July 2002. Geneva: World Health Organization; 2002.

2. Laurenti R, Mello-Jorge MHP, Gotlieb SLD. Perfil epidemiológico da morbimortalidade masculina. Ciênc Saúde Colet. 2005; 10:35-46.

3. Azambuja MIR, Foppa M, Maranhão MFC, Achutti AC. Impacto econômico dos casos de doença cardiovascular grave no Brasil: uma estimativa baseada em dados secundários. Arq Bras Cardiol. 2008; 91(3):163-171.

4. Schmidt MI, Duncan BB, Azevedo e Silva G, et al. Chronic non-communicable diseases in Brazil: burden and current challenges. Lancet. 2011; 377(9781):1949-1961.

5. Lee IM, Shiroma EJ, Lobelo F, et al. Effect of physical inactivity on major non-communicable diseases worldwide: an analysis of burden of disease and life expectancy. Lancet. 2012; 380(9838):219-229.

6. Kesaniemi YK, Danforth EJr, Jensen MD, et al. Dose-response issues concerning physical activity and health: an evidence-based symposium. Med Sci Sports Exerc. 2001;33(6):S351-358.

7. Simmons RK, Griffin SJ, Steele R, Wareham NJ, Ekelund U. Increasing overall physical activity and aerobic fitness is associated with improvements in metabolic risk: cohort analysis of the ProActive trial. Diabetologia. 2008; 51(5):787-794.

8. Smith DT, Carr LJ, Dorozynski C, Gomashe C. Internet-delivered lifestyle physical activity intervention: limited inflammation and antioxidant capacity efficacy in overweight adults. J Appl Physiol. 2009; 106(1):49-56.

9. Lau DC, Dhillon B, Yan H, Szmitko PE, Verma S. Adipokines: molecular links between obesity and atheroslcerosis. Am J Physiol Heart Circ Physiol. 2005; 288(5):H2031-2041.

10. Francisco G, Hernandez C, Simo R. Serum markers of vascular inflammation in dyslipemia. Clin Chim Acta. 2006; 369(1):1-16.

11. Church TS, Barlow CE, Earnest CP, et al. Associations between cardiorespiratory fitness and C-reactive protein in men. Arterioscler Thromb Vasc Biol. 2002; 22(11):1869-1876.

12. Ford, ES. Does exercise reduce inflammation? Physical activity and C-reactive protein among U.S. adults. Epidemiology. 2002; 13(5):561-568.

13. Aronson D, Sella R, Sheikh-Ahmad M, et al. The association between cardiorespiratory fitness and C-reactive protein in subjects with the metabolic syndrome. J Am Coll Cardiol. 2004; 44(10):2003-2007.

14. Colbert LH, Visser M, Simonsick EM, et al. Physical activity, exercise, and inflammatory markers in older adults: findings from the Health, Aging and Body Composition Study. J Am Geriatr Soc. 2004;52(7):1098-1104.

15. Elosua R, Bartali B, Ordovas JM, et al. Association between physical activity, physical performance, and inflammatory biomarkers in an elderly population: the InCHIANTI study.J Gerontol A Biol Sci Med Sci. 2005; 60(6):760-767.

16. Pitsavos C, Panagiotakos DB, Chrysohoou C, Kavouras S, Stefanadis C. The associations between physical activity, inflammation, and coagulation markers, in people with metabolic syndrome: the ATTICA study. Eur J Cardiovasc Prev Rehabil. 2005; 12(2):151-158.

17. Kullo IJ, Khaleghi M, Hensrud DD. Markers of inflammation are inversely associated with VO2 max in asymptomatic men. J Appl Physiol. 2007; 102(4):1374-1379.

18. Steene-Johannessen J, Kolle E, Reseland JE, Anderssen SA, Andersen LB. Waist circumference is related to low-grade inflammation in youth. Int J Pediatr Obes. 2010; 5(4):313-319.

19. Marques-Vidal P, Bochud M, Bastardot F, et al. Levels and determinants of inflammatory biomarkers in a Swiss population-based sample (CoLaus study). PLoS One. 2011; 6(6)e21002:1-8.

20. Martinez-Gomez D, Gomez-Martinez S, Ruiz JR, et al. Objectively-measured and selfreported physical activity and fitness in relation to inflammatory markers in European adolescents: the HELENA Study. Atherosclerosis. 2012; 221(1):260-267.

21. Azevedo MR, Horta BL, Gigante DP, Victora CG, Barros FC. Factors associated to leisuretime sedentary lifestyle in adults of 1982 birth cohort, Pelotas, Southern Brazil. Rev Saude Publica. 2008; 42(Supp1 2):70-77. 
22. Verdaet D, Dendale P, De Bacquer D, et al. Association between leisure time physical activity and markers of chronic inflammation related to coronary heart disease. Atherosclerosis. 2004; 176(2):303-310.

23. Craig CL, Marshall AL, Sjostrom M, et al. International physical activity questionnaire: 12-country reliability and validity. Med Sci Sports Exerc. 2003; 35(8):1381-1395.

24. Mielke GI. Comportamento sedentário em adultos. 2013. 149 f. Dissertação (mestrado) Programa de Pós Graduação em Epidemiologia, Universidade Federal de Pelotas, Pelotas. 2013.

25. Balke B, Ware RW. An experimental study of physical fitness of Air Force personnel. U S Armed Forces Med J. 1959; 10(6):675-688.

26. Nazmi A, Oliveira IO, Victora CG. Correlates of C-reactive protein levels in young adults: a population-based cohort study of 3827 subjects in Brazil. Braz J Med Biol Res. 2008; 41(5):357-367.

27. Arsenault BJ, Cartier A, Cote M, et al. Body composition, cardiorespiratory fitness, and lowgrade inflammation in middle-aged men and women. Am J Cardiol. 2009; 104(2):240-246.

28. Wilund KR. Is the anti-inflammatory effect of regular exercise responsible for reduced cardiovascular disease? Clin Sci (Lond). 2007; 112(11):543-555.

$$
\begin{array}{r}
\text { Endereço para Correspondência } \\
\text { Carlos Marcelo Cuello Moreno } \\
\text { Endereço: Rua Luis de Camões, 635, } \\
\text { Pelotas - RS } \\
\text { CEP: } 96055-630 \\
\text { Telefone: (53) 3273.2752 } \\
\text { E-mail: marckzelo@hotmail.com }
\end{array}
$$

